\title{
Exploring the System Determinants Associated with Senior Women's Access to Medical Care in Rural Bangladesh
}

\author{
Mohammad Hamiduzzaman ${ }^{1}$ (D) Anita De Bellis ${ }^{2} \cdot$ Wendy Abigail $^{2}$
}

Accepted: 25 July 2021 / Published online: 2 August 2021

(C) The Author(s), under exclusive licence to Springer Science+Business Media, LLC, part of Springer Nature 2021

\begin{abstract}
Senior women's access to medical care in rural Bangladesh is a major public health concern. This study aims to explore the system determinants that impact on rural senior women's utilization of healthcare. Following a qualitative critical social research design, healthcare staff and senior women living in three rural villages of Bangladesh were approached to participate in face-to-face audio-recorded interviews. A total of 11 staff and 25 senior women were interviewed with questions about health policy, healthcare services and management of aged care. Data was analyzed using a blend of critical discourse and thematic analysis methods. Several healthcare system determinants were identified that were complex and cross-sectional. Three major themes emerged from the system determinants: legal framework of aged care; inadequate healthcare support; and professional knowledge and skills of healthcare staff that led the rural senior women to avoid or delay access to hospitals and clinics. The findings revealed that a lack of health focus and professional skills among healthcare staff can be considered as critical. This study recommends that policy and organizational changes are made to improve the women's access to rural hospitals and clinics in Bangladesh.
\end{abstract}

Keywords System determinants $\cdot$ Rural senior women $\cdot$ Access to medical care Bangladesh

Mohammad Hamiduzzaman

khoka.hamiduzzaman@newcastle.edu.au

1 Research Fellow, College of Health, Medicine \& Wellbeing, The University of Newcastle, Newcastle, New South Wales, Australia

2 Senior Lecturer, College of Nursing \& Health Sciences, Flinders University, Adelaide, South Australia, Australia 


\section{Introduction}

Providing healthcare to senior women is not a part of everyday clinical practices for healthcare staff in rural and remote clinics and hospitals of low and lowermiddle income countries including Bangladesh. In Bangladesh, senior women are generally defined as being 60 years of age or older with current estimates at 7.3 million where approximately $73 \%$ live in rural villages (BBS, 2015). It is expected that the population of senior women will increase to 24.1 million by 2050 (BBS, 2015). Rural senior women's access to medical care encompasses preventive measures, regular visits to doctors, diagnosis, timely and appropriate treatment and rehabilitation, considering the physical and psychosocial care needs, which all have an effect on their functional and intellectual capacity and abilities to perform daily living activities (Princeton, 2015; Rahman et al., 2020). In clinics, hospitals and at the community level in Bangladesh, there are medically trained clinicians (i.e. doctors, nurses, pharmacists and pathologists) and semi-qualified care providers (i.e. paraprofessionals and community health workers) who provide direct care for older adults (Ahmed et al., 2011). Substantial variations in the proportion of physicians and nurses between rural and urban areas has been identified indicating that; while there were 18 doctors and 6 nurses allocated per 10,000 people in urban areas, only one doctor and one nurse were allocated for the same population size in rural areas (Ahmed et al., 2013). Low standards in the management of care and health conditions of rural senior women in clinical settings has also been identified (Hamiduzzaman et al., 2018a, 2018b).

Rural senior women with complex care needs are a heterogeneous group, with diseases and health conditions including heart disease, diabetes, hypertension, arthritis and isolation that vary by comorbidities, limitations and the need for medical care (Hossen, 2010; Rahman et al., 2020). Medical conditions experienced by these women tend to persist for longer periods of time than for other groups, for instance, the average duration across all diseases for the women is 76 months which is higher than the average duration of an ailment (i.e. 39 months) at the national level (BBS, 2012). This cohort with high needs, experience less healthcare access than any other population group, and as such represent a challenge for rural healthcare in Bangladesh. More specifically, only one woman in every 1,000 seeks care at local clinics and hospitals (BBS, 2012). While a few studies suggest that rural senior women have poor health and are less likely to access medical care, other studies have shown the women to be to attend preventive care and hospital, as well as not completing prescribed treatments (Biswas et al., 2006; Hamiduzzaman, 2018; Hossen \& Westhues, 2011, 2012). Some studies demonstrate that this lack of access to clinics and hospitals and rural senior women's unmet needs persist because of health system determinants (Biswas et al., 2006; Hamiduzzaman et al., 2016; Hossen et al., 2013).

There are a range of system determinants that have a potential impact on healthcare utilization by rural senior women, especially at the local level. For instance, a lack of aged care services and resources impacts negatively on the women where there is inadequate understanding of geriatric care among 
healthcare staff. (Hamiduzzaman et al., 2017; Iecovich \& Carmel, 2009). In addition, poor management of care, including long waiting periods in seeking care; and the inability of senior women to access health information and medical care in a timely manner results in higher costs of treatment (Ahren \& Hine, 2015). It is, therefore, assumed that quality care management for them in clinical settings that ensures the best outcomes for the women is yet to be realized. As rural senior women age with increasing frailty as the 'fourth age', as defined by Higgs and Gilleard (2015), they may experience further difficulties in accessing healthcare as they lose their autonomy in decision making and the capacity to navigate the clinics or hospitals alone. Despite previous research on self-reported senior women's access to healthcare in Bangladesh, little is known about the impact of health system determinants especially from the healthcare staff perspectives. This study attempts to explore this gap to: (i) investigate the system determinants and their complex relationships in relation to their impact on rural senior women's access; and (ii) understand the system determinants from the perspectives of both the healthcare staff and the rural senior women groups.

\section{Methods \& Materials}

This study is a qualitative exploration of the views of healthcare staff who provided care and rural senior women who received care in rural Bangladesh. A critical social research was conducted involving a blended critical social theory, semi-structured interviews with the participants and a critical thematic discourse analysis of the data.

The principles and dimensions of the healthcare sphere used in this study were developed using a blended critical social theoretical framework (Hamiduzzaman, 2018). The healthcare sphere was developed using the principles of emancipation and recognition; the domains of Habermas' Theory of Communicative Action (i.e. objective and subjective worlds); and Honneth's Theory of Recognition and Misrecognition (i.e. legal framework) (Habermas, 1984, 1989; Honneth, 2001, 2007). The healthcare sphere consists of two dimensions namely: (a) the institutionalization of care; and (ii) the rights of rural senior women and the responsibilities of healthcare staff (Hamiduzzaman, 2018, 2020, 2020). The first dimension of institutionalized care consists of various components such as legal factors, the availability of healthcare services and the management of care in hospital settings. The rights of these women and the responsibilities of healthcare staff concern the quality of healthcare access and include professional education and knowledge, as well as power attributes (Hamiduzzaman, 2018). These dimensions provided directions for the assessment of the quality of care management and were applied in the analysis and discussion sections to explain the systemic factors and issues impacting on rural senior women's access to medical care in Bangladesh.

After obtaining permission from the Directorate General of Health Services for recruitment and ethics approval from the Social and Behavioral Research Ethics Committee at the University (Project No. 6705), healthcare staff working in community clinics and hospitals and the senior women living in three villages of Tuker Bazar Union of Sylhet district were approached. There were three inclusion criteria 
in the recruitment of healthcare staff that included: (i) the staff worked in the provision of healthcare in Sylhet Sadar Upazila; (ii) had experience with providing care to rural senior women; and (iii) were willing to participate in a face-to-face interview. The inclusion criteria for recruiting rural senior women included: (i) they were aged 60 years and over and lived in the rural region; (ii) able to access local and/or distant medical services; (iii) able to provide informed consent; and (iv) willing to participate in face-to-face interview.

The Upazila Health Coordinator discussed the study during staff meetings and 11 healthcare staff confirmed their interest, and of those, there were three doctors, three pharmacists, one public health inspector and four healthcare assistants. Rural senior women were formally invited by the public health inspector to join in yardgatherings where information about the study was read out. Of the 39 women who attended the gatherings and responded, 14 were excluded because of age restrictions, permission requirements not being met from family and/or adverse health conditions. As such, 25 senior women participated in a face to face interview. The audiorecorded semi-structured interviews were conducted with the participants after obtaining written consent from them. Each interview included open ended questions and relevant prompts that directed discussions focused on healthcare policy, system and services and management of care in clinical settings. Interviews were conducted in Bangla (the native language) at a place and time convenient for the participants and were transcribed and translated into English.

The data coding and analysis conducted used a critical thematic discourse analysis method developed for the study. The thematic analysis focused on the exploration and description of the surface reality, while the critical discourse analysis improved the knowledge of discourses and dialectical relationships emphasizing the relative power of words and concepts. (Braun \& Clarke, 2006; Fairclough, 2013). Qualitative data analysis software NVivo 11.0 was used to facilitate the analysis process that involved six steps: (i) familiarity with the data and automated coding; (ii) each potential factor and action was labelled as open code; (iii) selective coding was conducted based on blended critical social theory and clustering of the codes into nodes; (iv) the second and third authors reviewed the codes and nodes were regular discussions reviewed the sub-themes and themes; and (v) the sub-themes were crosschecked against the data [revisited and reviewed the codes and nodes by all authors against factors, events and actions emerged from data to ensure consistency among the data, the categories, the sub-themes and the themes] and with naming of the subthemes and themes reviewed; and (vi) the theoretical dimensions of the healthcare sphere were applied to the themes and sub-themes (Hamiduzzaman, 2018).

\section{Findings}

Three major themes of the perspectives of the healthcare staff and rural senior women emerged with several sub-themes including: (i) the legal framework of aged care; (ii) inadequate healthcare support; and (ii) the professional knowledge and skills of healthcare staff. Each of the themes is presented with extracts that represent the voice of the participants in this research. 


\section{The Legal Framework of Aged Care}

The main healthcare system theme identified concerned the legal framework that influenced rural senior women's access to medical care. This legal framework involved a combination of constitutional rights, healthcare policies and programs and the protection of human rights in clinical settings.

The healthcare staff discussed in detail the ineffectiveness of constitutional laws in meeting healthcare needs of older adults, women in particular. Access to healthcare emerged in the discussions of most healthcare staff as one of the written constitutional rights for all citizens. However, this was not actualized or practiced in the rural healthcare system due to the needs of senior women receiving little attention from policy makers or health system authorities in upholding their right to healthcare access. The lack of knowledge among the women regarding their constitutional and legal rights also emerged in their discussions where policy makers relegated their voice to one of low importance. For example:

They have constitutional rights, but these all are in writings or in books. In practice, they do not have anything because we obey the decision made by our higher authorities. As you know, we are progressing in maternal and child health ... and have limited scope to ask them to change the focus (Healthcare staff Shamim; p.84; line no. 2-3).

I heard about the constitution, but do not know whether there is anything written about us as I can't read. No one told me about it ... who will be telling the government for me? (Rural senior woman Fatima; p.236; line no. 11-12).

The ignoring of senior women's healthcare needs by policy makers led to challenges for the women when visiting clinics or hospitals in terms of unequal access.

According to the majority of healthcare staff, the national healthcare policies and programs were developed focusing on the needs of maternal and child healthcare without consideration for the health and healthcare needs of senior women. There were several public health programs provided including health education and basic door-to-door care services for promoting maternal and child care, whilst there were no programs identified by most participants regarding the women's healthcare promotion. The following exemplars highlight this issue.

The government does not provide any healthcare services for rural senior women living in this area. We are guided by the Civil Surgeon office to provide care to maternal and child care. There is nothing written in healthcare policies and programs about these women as I know ... Current health care services and practices concentrated on pregnant women and their infants (Healthcare staff Panna; p.34; line no. 11-14).

I visited the community clinic and family welfare center few months ago as they are close from my home. I waited there for one hour and returned home without any medications. They suggested me to visit XXX hospital as they only provide care to children and pregnant women. (Rural senior woman Sofia; p.205; line no. 15-17). 
Some women described an annual eye care program operated by a voluntary organization, but according to the staff and women participants, the major focus of the national healthcare policy and local healthcare system was on maternal and child health resulting in a lack of focus on senior women's access to medical care.

The rejection of responsibility by healthcare staff was identified by participants as another barrier for the women accessing medical care. When these women reported a high need of healthcare, some healthcare staff blamed the women for not visiting them such as described in the following extract:

In health sector, we are trying our best to establish human rights for any person even for women or young. If they come to us, we can give them best support. But these women have different perspective from me (Healthcare staff Tarek; p.121; line no. 4-6).

In contrast, the women described the power of healthcare staff in medical practices as discriminatory, for example:

In public hospitals, we are nothing in front of the doctors and nurses. They will provide you care when they want to, you can't say anything. If someone wants to tell something, there is a chance of not being treated. However, there are some good doctors who called me ma [mother] and I always try to visit them (Rural senior woman Salma; p.171; line no. 3-4).

This meant that some healthcare staff lacked interest in establishing easier access for the women and were, as such, not accountable for providing healthcare and health information to these women. This lack of accountability was evident in the discourses and, additionally, the constitutional protection was not reflected in the national healthcare policies and programs appropriately. This situation was further compounded by the lack of provision of medical services to rural areas.

\section{Inadequate Healthcare Support}

The second major theme of inadequate healthcare support was identified by both the healthcare staff and rural senior women. The participants identified poor access for the women to medical care related to the lack of and unavailability of healthcare services, the high cost of medical care, low staffing levels, an inadequate supply of equipment and medication, the long waiting times for treatment and an inadequate referral system.

According to the participants, there were limited healthcare facilities and services for the women. There were three public clinics, hospitals and several private clinics identified by healthcare staff in the region, however, the women described visits to the local community clinics, but most of them discussed access to the public hospital outside the region.

Tuker Bazar Union did not have community clinic before. There is a new clinic established. There are eight tea gardens in the union. But the healthcare centers of this union are poorly organized (Healthcare staff Asma; p.2; line no. $1-3)$. 
There are no public healthcare facilities in our village. I have to go for a long way for accessing healthcare centers. There are few private medicals, but they are located outside this village (Rural senior woman Shakira; p.159; line no. 22-23).

According to the rural senior women, healthcare services were only available for one day per week in the local clinics and the reasons for the lack of services were described by the staff and women differently.

There is a huge pressure of patients in the hospitals. ... we can provide support for only a small proportion of population ... (Healthcare staff Tarek; p.118; line no. 8-10).

... treatments are not available in the local clinics except XXX hospital. If you have money, there are treatments for you. As I am poor, I cannot access private healthcare (Rural senior woman Rawson; p.224; line no. 21).

The limited number of clinics and hospitals and lack of services in the region resulted in the exclusion of these women from accessing medical services. The costs incurred when seeking medical treatment were identified by both participant groups as a barrier. However, there were dissimilar views expressed during the discussions by healthcare staff and the women in relation to the cost of medical care. While staff reported free treatment in public clinics and hospitals, most of the women reported the cost of medical care prohibitive due to the costs involved in travelling to hospitals, payment of hospital bills and the cost of medications in both public and private hospitals. These costs were described by the some of the women in the following extracts:

... if you know a driver, you can ask him to take you to hospital. They will take you though they charged you double. There are no public transports in this road so that we have to use privately owned vehicles. It costs more (Rural senior woman Sofia; p.204; line no. 25-27).

In private hospital, they ask for lots of money. No-one can get treatment without money. Anywhere you go, you have to spend money. Who will give me the money? ... Without money, they will never give you treatment. Doctors ask for 500-600 BDT from me for each visit. Sometimes they ask for more money than the actual charge (Rural senior woman Anwara; pp.178-179; line no. 24-25 (p.178) and 23-24 (p.179)).

They do not charge money directly, but they always suggested for different pathology tests. This is happening in public hospital. Undertaking these tests requires a lot of money. While I can do a test by 10 BDT in any laboratory, I need to spend 50 BDT as doctor prescribed me to go there. Is this possible to pay this huge amount for a poor person like me? Now I need to see a doctor for my eyes, but I cannot go there, as I need 2000 BDT (Rural senior woman Shakira; p.160; line no. 21-25).

According to the women, the high cost in seeking care forced them to avoid medical treatment, or sometimes having to leave the hospital in the middle of treatment as they could not afford to complete it. 
Both participant groups indicated a shortage of doctors, nurses and other staff in the clinics and hospitals that further impacted on the women's access to services and treatment. The staff shortage, as reported by the participants, was due to the low numbers of medically trained clinicians and gerontology specialists in regional clinics and hospitals.

We do not have enough staff. Though one staff is responsible to provide healthcare support for 4,000 people nationally, I am providing support for more than 12 thousand people in this area. ... It is difficult for me to manage such a big population. We cannot provide enough support due to inadequate staff. I also must visit other community clinics (Healthcare staff Abul; pp.20-21; line no. 18-20 (p.20) and 1-2 (p.21)).

In addition, an absence of doctors and nurses in the clinics and hospitals was identified by the women as a factor in their timely healthcare use. For example:

I did not feel comfortable in public hospital. In public hospital, they told me to sit on floor and they did not give me any beds. I went there at 10 am and one doctor came to see me at $5 \mathrm{pm}$. There were no other doctors for this general unit (Rural senior woman Sharifa; p.150; line no. 19-22).

If doctor is available, it takes 2 hours. However, if you do not get support from doctor or nurse, you must wait for a long time. Doctor used to come late in the hospital. They came into the hospital one or two hours late. If doctors do not come at right time, you must wait (Rural senior woman Sofia; p.205; line no. 21-23).

The low number of healthcare providers was also reported by the rural senior women to include a lack of female doctors in regional clinics. While some staff described the prevalence of accessing female doctors had been changing the women's preference for female doctors and the lack of female doctors emerged in their discussions.

Female doctors are good for me as I can share everything with them. These doctors can understand my problem. I do not visit male doctor. I have been visiting female doctors for the last four years. Female doctor from a private hospital did a surgery for me. I feel shy to share my problems with male doctors. Is that possible to share everything to male doctors? Experienced and skilled doctors are all male. I went to male doctors mostly (Rural senior woman Rinku; p.291; line no. 15-19).

Whilst the participants reported low numbers of clinicians, including female doctors, the women also discussed their experiences of a lack of doctors in hospitals that contributed to them not using or accessing medical care.

Most of the participants reported restricted access to adequate healthcare for the women due to a lack of equipment, supplies and medications in local clinics. Some staff stated that there was a yearly allocation of medical equipment and a monthly supply of medications, but the supply was identified as irregular and insufficient. For example: 
We cannot conduct any tests for the patients. We do not have equipment even at Upazila level. If they require CT scan or MRI scan, they need to travel to district level hospitals at city.... There is a lack of medication supply in the healthcare centers. We should acknowledge that there are bureaucratic problems or rigidity in getting adequate and regular medication supply. Thus, the medical officer gives a prescription for them and asks them to collect these medications from family welfare center or to buy from local pharmacies (Healthcare staff Tarek; pp.112-117; line no. 18-20 (p.117) and 12-15 (p.112)).

Most of the women reported that medications were not available in the hospitals and community clinics, where healthcare assistants working in public hospitals either sold medicinal stock to pharmacies or took the drug stock from the hospital for their own dispensaries.

Medications are available in the pharmacies. When I visited a local clinic, doctor prescribed me several medications and suggested me to buy these from a local pharmacy. I heard that the doctor I visited was the owner of this pharmacy (Rural senior woman Tamanna; p.306; line no. 9-11).

This lack of equipment and medications, together with the lengthy waiting times in seeking medical health services, presented problems that deterred the women from accessing medical care. It was acknowledged by healthcare staff and the women that seeking out-patient and in-patient medical care involved long waiting times. Although most staff blamed the huge number of patients, one staff described the absence of doctors during the scheduled consulting hours.

Doctors usually see patients up to $2.00 \mathrm{pm}$, which supposed to be started from $8.00 \mathrm{am}$. However, I did not see any doctor come in the hospital at right time. Doctors used to come to the hospital after $11.00 \mathrm{am}$. People buy ticket in the early morning and wait for doctors (Healthcare staff Suchona 5; p.52; line no.1-4).

Some women blamed the system that created an additional delay in seeking care from hospitals, as described in the following extracts:

I may get some free medicines from XXX medical and this will help me. However, it takes a day to visit XXX medical and this will ruin my business. ... You must be in a waiting line for buying ticket. After having ticket, you need to wait for doctor's appointment. After getting prescription from doctor, you must wait for medicines. For this reason, I do not like to visit hospital. Same conditions are in private hospital too (Rural senior woman Sofia; p.205; line no. 13-17).

I am waiting for three days for my bone test. I am going to the hospital each day and they are asking me to come next day at every time (Rural senior woman Jamila; p.314; line no. 15-17).

Prolonged waiting times when seeking care had a negative impact on these women's timely and regular access to medical health services, which was further compounded by an ineffective referral system. 
The ineffective referral system was identified by the healthcare staff as a barrier to providing complete care to senior women at the district hospital level. Due to a lack of coordination between public and private clinics and hospitals, some staff reported a reluctance in referring the women to regional private hospitals. For example:

We can refer the patients from Union level to Upazila level to District hospital. We try our best to keep the patients in the public hospital. We do not like to refer the patients to the private hospitals. If any patient wants to go for private hospitals for better care, they can go there. I think we do not have coordination with private hospitals. If the patient is in critical condition, we can refer the patient to the higher level such as XXX Medical University or XXX medical college at capital city. We do not want to depend on the private hospitals (Healthcare staff Tarek; p.115; line no. 4-10).

Even though health services and practices had been established in the clinics and hospitals for the treatment of the population, these did not match the healthcare needs of rural senior women. There was a lack of aged care related services, staff, equipment and medications, as well as high costs and long waiting times when seeking care. The care provided was also identified by the staff and the women as not appropriate due to a lack of academic and professional knowledge by staff about gerontology and the healthcare needs of older adults.

\section{Professional Knowledge and Skills of Healthcare Staff}

Healthcare access was described by the both participant groups in terms of the doctors and nurses' lacking professional knowledge, having inadequate communication skills, and favoring affluent people. Two issues were identified during the discussions with the staff regarding their understanding of rural senior women's needs and healthcare support: (a) most of the staff entered the healthcare sector without adequate or formal geriatric education and/or training; and (b) the medical education or training received by the staff was related to maternal and child health. Examples of this included:

Discrimination can be found in another aspect. Rural senior women are in requirement of advance healthcare support, which is unavailable in our hospitals. Doctors and nurses do not have education and knowledge about aged care (Healthcare staff Tapan; p.104; line no. 19-21).

I have participated in few basic training courses on Expanded Program on Immunization (EPI) and maternal healthcare. I did not receive any training for older women's healthcare. I did not even hear about any training on these women's healthcare services (Healthcare staff Panna; p.26; line no. 10-13).

This lack of academic knowledge was considered by the heath staff as a reason for inadequate care for the women in local clinics and hospitals. Professional incompetency of staff was also reported by the women about negative interactions between clinicians and the women. 
Healthcare staff reported that rural senior women lived self-regulated lives within a family structure who did not converse much, except to their family members. The staff stated this resulted in incompetence among these women in communicating with doctors and nurses. Most of the women reported differently in that the doctors and nurses did not listen to them, as stated in the following extracts:

But sometimes they do not like to hear from me. They said that we know better than you, and you have no need to say anything about your diseases. They just give me a prescription without knowing anything about my physical problems. These medications do not work for my diseases. They do not like to listen me, and they asked me to stop talking about my health problems (Rural senior woman Amena; p.139; line no. 3-7).

I was near to death and my relatives admitted me into XXX hospital.... when my aunt gave her details to the hospital authority, they took me to the general beds. After few hours, four doctors came to see me and asked the attending nurse why she admitted me for this bed. For this reason, she [nurse] transferred me to another small room. I heard one patient just died in this bed. I become afraid. On this time, I was hearing everything, and I could not talk. I was there for one month (Rural senior woman Shakira; p.162; line no. 15-21).

The lack of communication and inadequate assessments by doctors and nurses led the women to believe that healthcare staff and hospitals were not there for them. Furthermore, there was a power relationship during clinical interactions identified by the participants. According to some staff, healthcare for senior women was mostly mediated by their male family members due to negative behaviors of the doctors and nurses towards the women. The rural senior women added that the clinical encounters were dominated by the doctors and nurses and they were not treated well. For example:

... doctors and nurses used to behave badly to them. Many poor women came to me and said that the doctors and nurses are shikkhito pagol [educated psycho] (Healthcare staff Selim; p.65; line no. 9-10).

They [doctors and nurses] do what they want to do. This is not related with my satisfaction or dissatisfaction. Will they listen to me? Why they listen to a poor and old woman like me? If I cried for the whole day in front of them, they will never listen to me. They will do according to the rules of their hospital (Rural senior woman Fatema; p.189; line no. 4-7).

These experiences of negative behaviors towards the rural senior women and the power differences reflected in the women's decisions to use or not use medical care.

These poor relationships were also compounded by the women's perception of nepotism by the doctors and nurses when providing care to them. The favoring of affluent people by healthcare staff was identified by the women as being a barrier to accessing care. Some staff also indicated there was a close relationship between the economic status of the women and access to medical care. For example:

Senior women receive a range of healthcare services when the family is rich .... Their importance is largely depending on the socioeconomic status of the 
family. I cannot tell you exactly why it happens, but it is a little bit of frustration. Where do they get a lot of money? (Healthcare staff Asma; p.2; line no. 18-21).

Some women also described that kinship played a strong part in them receiving quick and quality treatment. One woman stated that she did not receive quality care because she had no relatives working in the clinics:

People known to the doctors and nurses receive good behavior from them. As no-one knows me in the hospital; they do not behave well with me (Rural senior woman Jamila; p.313; line no. 13-14).

This discrimination of patients based on their financial situation and kinship practices created a negative perception and impacted on rural senior women in accessing medical care.

In summary, there were three major areas of the health system that impacted on whether the rural senior women accessed medical care or not including: an ineffective legal framework for aged care; inadequate healthcare support; and a lack of professional knowledge and skills of healthcare staff. Although the staff and women had similar views, their perspectives sometimes differed slightly, but all participants agree that the access to medical care for senior women was far from optimal.

\section{Discussion}

Medical care access needs of senior women are greater than other population groups due to these women being more prone to chronic and comorbid conditions (Ahern \& Hine, 2015). However, the determinants of the women's access to medical care are evident within a complex healthcare system, as identified in other studies (Hamiduzzaman, 2018; Hossen, 2016). Policy changes are required, however when considering policy and program changes, understanding the determinants within healthcare system identified in this study are useful. Consideration as to what is happening in clinical structures and practices from the two dimensions of the healthcare sphere and of the critical social theoretical framework can be considered when policy is being formulated.

Two areas emerged in the dimension of institutionalized care, from the views of participant groups to recognize the healthcare needs of rural senior women in accessing medical care: (i) the legal framework; and (ii) the management of care. The institutionalized care dimension starts with an analysis of the legal focus of healthcare planning and clinics/hospitals as a determinant and how these take place in a stratified society. While most senior women live in rural villages with multiple morbidities and chronic conditions, the National Healthcare Policy 2011 of Bangladesh, as in other low and lower-middle income countries, has no specific plans or programs to provide medical assistance for them (Akseer et al., 2017). The focus on maternal and child healthcare in healthcare laws and policies influence the scope of clinics/hospitals to meet their medical care needs. The focus on child and maternal health is primarily due to concerns of maternal and child mortality by the World 
Health Organization (Haider et al., 2017). This result in the development of local clinics/hospitals and services focusing on the needs of children and pregnant women in most of the low-income countries including Bangladesh (Berman et al., 2016). The findings of Khanam et al. (2011) indicate that any policy effort, along with aged care programs, to reorganize healthcare should consider the needs of senior women and recognize them as 'human beings' and 'patients', because these women are often powerless and restricted when accessing medical care.

The capacity and performance of clinics and hospitals in the management of care for rural senior women can be explained within the dimension of institutionalized care (Hamiduzzaman et al., 2020a, 2020b; Princeton, 2015). The determinants, according to the women, of inadequate access to medical care; a lack of geriatric services, staff, equipment and medications; and lengthy waiting periods when seeking care are critical issues. This is reinforced by the healthcare staff who revealed that the local clinics and hospitals struggle due to the burden of overpopulation. In addition, the participant groups possessed different opinions concerning the costs of seeking care that was compounded by an ineffective referral system. Princeton (2015) and Hamiduzzaman (2018) state that a critical social explanation of the understanding of healthcare needs of an underprivileged person, such as rural senior woman, should be in the context of their functional, clinical, personal and social needs. The management of their care includes home care, safe travelling, skilled medical care, psychological services, therapies, social activities and rehabilitation (Brinda et al., 2015). This perspective of healthcare, along with the management of care, challenges the capacity of regional hospitals and clinics, especially in lowincome countries like Bangladesh, to provide holistic but cost-effective care to them (Ameh et al., 2014; Amin, 2017; Uddin \& Hamiduzzaman, 2009). Having a biomedical focus on healthcare in combination with a lack of services and resources, the high cost of care and poor referral systems causes general disadvantage; thus, well-articulated professional training for clinicians has the potential to increase their access to medical care.

The second dimension of the healthcare sphere concerns the responsibilities of healthcare staff in the management of care related to two areas namely: (i) a lack of professional knowledge about gerontology; and (ii) power attributes. The lack of medical education and training of staff on geriatrics and gerontology is a determinant of the relationship between the staff and the women in this study, and closely related to rural senior women's not accessing medical care. According to Stewart (2011) and Strasser et al. (2016), clinicians require sound medical knowledge about physiology, pathology, treatment options and professional ethics prior to the provision of geriatric care. Literature indicates that clinicians, especially in rural villages of lowincome countries, have a lack of professional competency in providing care to senior patients (Ahmed et al., 2011; Hamiduzzaman et al., 2020, 2020; Scott et al., 2016). This inadequate knowledge among healthcare staff and a lack of opportunity for geriatric education and training is indicated in other studies (Princeton, 2015; Terraneo, 2015). Ongoing education on gerontological care can assist healthcare staff to be responsive to an older person's healthcare needs through better understanding, skills and objective decision-making abilities. These skills are also important in diminishing power differences between senior persons and professionals. 
The dimension of responsibilities of healthcare staff also needs to include an understanding of clinical interactions and relationships between rural senior women and professionals in regard to power relationships. Less than optimal relationships and ineffective communication were identified in this study. While the staff identified the self-regulated life of these women as a factor, the women described the negative behaviors and patient classifications by doctors that encouraged them to rely on their male family members when communicating with healthcare staff (Hamiduzzaman et al., 2020). Inadequate relationships and missed interactions are deeply rooted in the socioeconomic classification of professions and the male dominance in medical practices, especially in low-income countries like Bangladesh (Hamiduzzaman et al., 2017, 2018a). Mutual trust is essential for sharing health information and encouraging regular visits to hospitals (Bergeron et al., 2018; Loewenson \& Simpson, 2017; Zhang et al., 2017). More work is required to assess whether the current healthcare workforce is prepared to care for the complex needs of rural senior women and to be aware of the socio-cultural aspects of the healthcare system and staff themselves negatively influence the access of the women to medical care.

One of the major strengths of this research was to apply the aspects of the healthcare system in order to explore the mediating impact of system determinants on rural senior women access to medical services. Moreover, this study also had a focus on the women themselves who are regarded as being in need of healthcare, vulnerable and with no voice. The identified health system determinants of an effective legal framework, a lack of medical training and professional values of healthcare staff, as well as power differences, all impacted on women's access to medical care in Bangladesh.

Among the limitations of this research, due to time restrictions in gathering data, was the lack of access to family members who accompanied the rural senior women when travelling and managing their healthcare. It must be acknowledged that due to some socioeconomic differences among different age and sex groups, explanations of healthcare access for the women might be different from a young adult and/or an older family member such as son, daughter and/or husband. Regarding the qualitative nature of this study, there were causal relationships among determinants, however, findings should be understood as context specific. Despite these limitations, this study contributes to the body of knowledge in this under researched area of healthcare in low-income countries highlighting the system determinants that impact on rural senior women in Bangladesh when accessing medical services.

\section{Conclusions}

This study considered perspectives of healthcare staff and rural senior women concerning the women's access to medical care. A combined critical and social insight into the impact of the health system determinants on access. The findings revealed a shared viewpoint on the scarcity of services and a lack of staff, medical equipment and medications. The care management factors identified by the participants were related to long waiting times, difficulties in seeking treatment, negative behaviors of healthcare staff and power differences. The system determinants of these women's 
access were related to the focus of healthcare policies and programs in Bangladesh, the inadequate medical education and training of healthcare staff, the burden of overpopulation and an inadequate referral system.

To improve access to healthcare for older women, all hospital, community and family-based strategies are important. Two key strategies include the redistribution of healthcare staff and resources in rural areas and the provision of on-going gerontological education for staff. The education needs to be targeted to the needs and expectations of senior women based on strategies that have been successful in other countries for promoting their access to medical services. Furthermore, the performance of healthcare staff in providing care such as education, skills and professionalism need to be challenged so that with better clinical education, training and standards of aged care, the possibility of the women's engagement in clinics and hospitals is promoted. Additionally, policy changes are required to encourage healthcare staff to advocate, negotiate and/or argue for improved healthcare rights of rural senior women healthcare right to contribute to policies and programs on the national agenda.

Acknowledgements The authors are thankful to the participants [healthcare staff and rural senior women] who provided their time and shared their healthcare providing and seeking experiences. They grateful to the Ministry of Health \& Family Welfare, Bangladesh for assisting in the recruitment of participants.

Authors' Contributions MH made substantial contributions to conception and design, acquisition of data, analysis and interpretation of data. Responsible for drafting the manuscript and revising it critically for important intellectual content. ADB and WA made substantial contributions to shaping the conception and design. Involved in drafting the manuscript and revising it critically for important intellectual content. All authors read and approved the final manuscript.

\section{Compliance with Ethical Standards}

Conflict of Interest The authors declare that they have no conflict of interest.

Ethical Approval All procedures performed in studies involving human participants were in accordance with the ethical standards of the Flinders University Social and Behavioural Research Ethics Committee (Project Number: 6075) and with the 1964 Helsinki declaration and its later amendments or comparable ethical standards.

Informed Consent Informed consent was obtained from all individual participants included in the study.

\section{References}

Ahern, A., \& Hine, J. (2015). Accessibility of health services for aged people in rural Ireland. International Journal of Sustainable Transportation, 9(5), 389-395.

Ahmed, S. M., Hossain, M. A., RajaChowdhury, A. M., \& Bhuiya, A. U. (2011). The health workforce crisis in Bangladesh: Shortage, inappropriate skill-mix and inequitable distribution. Human Resources for Health, 9(1), 3.

Ahmed, S. M., Evans, T. G., Standing, H., \& Mahmud, S. (2013). Harnessing pluralism for better health in Bangladesh. The Lancet, 382(9906), 1746-1755.

Akseer, N., Kamali, M., Arifeen, S. E., Malik, A., Bhatti, Z., Thacker, N., Maksey, M., D’Silva, H., Da Silva, I.C., \& Bhutta, Z. A. (2017). Progress in maternal and child health: How has South Asia fared? $B M J, 357, \mathrm{j} 1608$. 
Ameh, S., Gómez-Olivé, F. X., Kahn, K., Tollman, S. M., \& Klipstein-Grobusch, K. (2014). Predictors of health care use by adults 50 years and over in a rural South African setting. Global Health Action, $7(1), 24771$.

Amin, I. (2017). Perceptions of successful aging among older adults in Bangladesh: An exploratory study. Journal of Cross-Cultural Gerontology, 32(2), 191-207.

BBS. (2012). Gender statistics of Bangladesh. Bangladesh Bureau of Statistics. Ministry of Planning.

BBS. (2015). Adjusted population and housing census. Bangladesh Bureau of Statistics, Ministry of Planning.

Bergeron, C. D., Hilfinger Messias, D. K., Friedman, D. B., Spencer, S. M., \& Miller, S. C. (2018). Involvement of family members and professionals in older women's post-fall decision making. Health Communication, 33(3), 246-253.

Berman, P., Requejo, J., Bhutta, Z. A., Singh, N. S., Owen, H., \& Lawn, J. E. (2016). Countries' progress for women's and children's health in the Millennium Development Goal era: The Countdown to 2015 experience. BMC Public Health, 16(2), 1-4.

Biswas, P., Kabir, Z. N., Nilsson, J., \& Zaman, S. (2006). Dynamics of health care seeking behaviour of elderly people in rural Bangladesh. International Journal of Ageing and Later Life, 1(1), 69-89.

Braun, V., \& Clarke, V. (2006). Using thematic analysis in psychology. Qualitative Research in Psychology, 3(2), 77-101.

Brinda, E. M., Kowal, P., Attermann, J., \& Enemark, U. (2015). Health service use, out-of-pocket payments and catastrophic health expenditure among older people in India: The WHO Study on global AGEing and adult health. Journal of Epidemiology and Community Health, 69(5), 489-494.

Fairclough, N. (2013). Critical discourse analysis and critical policy studies. Critical Policy Studies, 7(2), 177-197.

Habermas, J. (1984). The theory of communicative action (Vol. 1). Beacon press.

Habermas, J. (1989). The theory of communicative action. Lifeworld and system: A critique of functionalist reason (Vol. 2). Boston: Beacon press.

Haider, M. R., Rahman, M. M., Moinuddin, M., Rahman, A. E., Ahmed, S., \& Khan, M. M. (2017). Impact of maternal and neonatal health initiatives on inequity in maternal health care utilization in Bangladesh. PloS One, 12(7), e0181408.

Hamiduzzaman, M. (2018). The world is not mine: Factors and issues of rural elderly women's access in medical care services in Bangladesh. $\mathrm{PhD}$ Thesis. Adelaide: Flinders University.

Hamiduzzaman, M., De Bellis, A., Kalaitzidis, E., \& Abigail, W. (2016). Factors impacting on elderly women's access to healthcare in rural Bangladesh. Indian Journal of Gerontology, 30(2), 235-260.

Hamiduzzaman, M., De Bellis, A., Abigail, W., \& Kalaitzidis, E. (2017). The social determinants of healthcare access for rural elderly women - A systematic review of quantitative studies. The Open Public Health Journal, 10(1), 244-266.

Hamiduzzaman, M., de Bellis, A., Abigail, W., \& Kalaitzidis, E. (2018a). Elderly women in rural Bangladesh: Healthcare access and ageing trends. South Asia Research, 38(2), 113-129.

Hamiduzzaman, M., De Bellis, A., Abigail, W., \& Kalaitzidis, E. (2018b). Social determinants of rural elderly women's healthcare access: A systematic review of qualitative literature. The Indian Journal of Social Work, 79(4), 469-496.

Hamiduzzaman, M. (2020). Self-reported seasonal symptoms and diseases and primary healthcare utilization among rural elderly women in Sylhet district. Bangladesh. Journal of UOEH, 42(2), $175-185$.

Hamiduzzaman, M., Kuot, A., Greenhill, J., Strivens, E., \& Isaac, V. (2020). Towards personalized care: Factors associated with the quality of life of residents with dementia in Australian rural aged care homes. PloS One, 15(5), e0233450.

Hamiduzzaman, M., Torres, S., Fletcher, A., Islam, M. R., \& Greenhill, J. (2020). Aging, care and dependency in multimorbidity: How relationships affect older Bangladeshi women use of homecare and health Services. MedRxiv. https://www.medrxiv.org/content/medrxiv/early/2020/06/22/2020.06. 19.20126078.full.pdf

Higgs, P., \& Gilleard, C. (2015). Rethinking old age: Theorizing the fourth age. Palgrave Macmillan.

Hossen, A. (2010). Bringing medicine to the hamlet: Exploring the experiences of older women in rural Bangladesh who seek health care. $\mathrm{PhD}$ thesis. Canada: Wilfrid Laurier University.

Hossen, M. A. (2016). Older women (user) perspective toward service delivery system of government hospital: A study on some upazila health complex of Bangladesh. Society \& Change, 10(1), 18-27.

Hossen, M. A., \& Westhues, A. (2011). Rural women's access to health care in Bangladesh: Swimming against the tide? Social Work in Public Health, 26(3), 278-293. 
Hossen, A., \& Westhues, A. (2012). In search of healing between two worlds: The use of traditional and modern health services by older women in rural Bangladesh. Social Work in Health Care, 51(4), 327-344.

Hossen, M. A., Westhues, A., \& Maiter, S. (2013). Coping strategies of older rural Bangladeshi women with health problems. Health Care for Women International, 34(12), 1116-1135.

Honneth, A. (2001). Recognition or redistribution?. Theory, Culture \& Society, 18(2-3), 43-55.

Honneth, A. (2007). Recognition as ideology. In: Brink, B.V.D., Owen, D. (eds.) Recognition and power: Axel Honneth and the tradition of critical social theory. Cambridge: Cambridge University Press.

Iecovich, E., \& Carmel, S. (2009). Differences in accessibility, affordability, and availability (AAA) of medical specialists among three age-groups of elderly people in Israel. Journal of Aging and Health, 21(5), 776-797.

Khanam, M. A., Streatfield, P. K., Kabir, Z. N., Qiu, C., Cornelius, C., \& Wahlin, А. (2011). Prevalence and patterns of multimorbidity among elderly people in rural Bangladesh: A cross-sectional study. Journal of Health, Population, and Nutrition, 29(4), 406-414.

Loewenson, R., \& Simpson, S. (2017). Strengthening integrated care through population-focused primary care services: International experiences outside the United States. Annual Review of Public Health, $38,413-429$.

Princeton, D. M. (2015). The critical theoretical perspectives and the health care system. Review of Arts and Humanities, 4(1), 72-79.

Rahman, M. M., Hamiduzzaman, M., Akter, S., Farhana, Z., Hossain, M. K., Hasan, N., \& Islam, M. N. (2020). Frailty indexed classification of Bangladeshi older adults' physio-psychosocial health and associated risk factors: A cross-sectional survey study. MedRxiv. https://assets.researchsquare.com/ files/rs-34134/v1/c5a2adf8-2e79-4677-8eef-8a2c4b572b8a.pdf

Scott, J., Revera Morales, D., McRitchie, A., Riviello, R., Smink, D., \& Yule, S. (2016). Non-technical skills and health care provision in low-and middle-income countries: A systematic review. Medical Education, 50(4), 441-455.

Stewart, J. (2011). Nutritional status of older patients admitted to hospital for surgery. British Journal of Community Nursing, 16(11), S18-S20.

Strasser, R., Kam, S. M., \& Regalado, S. M. (2016). Rural health care access and policy in developing countries. Annual Review of Public Health, 37, 395-412.

Terraneo, M. (2015). Inequities in health care utilization by people aged 50+: Evidence from 12 European countries. Social Science \& Medicine, 126, 154-163.

Uddin, M. N., \& Hamiduzzaman, M. (2009). Problems and prospects of decentralised rural healthcare system in Bangladesh. SUST Journal of Public Administration, 2(1), 47-75.

Zhang, X., Dupre, M. E., Qiu, L., Zhou, W., Zhao, Y., \& Gu, D. (2017). Urban-rural differences in the association between access to healthcare and health outcomes among older adults in China. BMC Geriatrics, 17(1), 151-161.

Publisher's Note Springer Nature remains neutral with regard to jurisdictional claims in published maps and institutional affiliations.

Mohammad Hamiduzzaman Dr Mohammad Hamiduzzaman is social epidemiologist and health services researcher, works as Research Fellow in the College of Health, Medicine \& Wellbeing at The University of Newcastle. He has completed his $\mathrm{PhD}$ about determinants of rural older womens access to healthcare and has extensive research work record in residential dementia care, personalised aged care, virtual healthcare support, clinical interactions, health services structuring, and health beliefs and behaviours. Major focus of his projects' is to improve physical and intellectual capacity of disadvantage people through public health interventions such as person-centered healthcare, health literacy, healthcare seeking behavioral tools and technology such as mobile app, website or social media. In this regard, he has theoretical knowledge and methodological skills and experience in leading and managing pharmacological and nonpharmacological healthcare projects in different regions of Australia. Expertise in using research software such as REDCap, SPSS and NVivo added value in his research management skill and capacity. Also he has skills in developing toolkit [i.e. VERILYConnect; \& Ageing Well in Harmony] that help people to be benefited from project outcomes. He is a co-investigator of two national research projects, funded by Australian Department of Health: (i) Harmony in the Bush: A Personalised Model of care for Dementia [Chief Investigator/AUD 1.5 Million]; (ii) VERILYConnect: Virtual 
Dementia Friendly Rural Communities [Project Coordinator \& Associate Investigator - South Australia/ AUD 1.7 million]. He is leading three seeding grant projects: (i) Data-Linkage project of older adults' allied healthcare use (The College Pilot Grant - AUD 15K); (ii) Person-centered clinical interactions of older adults (Flinders University Internal College Grant - Principal Investigator/AUD 15K); and (iii) COVID-19 Risk management strategies by older adults [Flinders University Internal College Grant - Principal Investigator/AUD 20K). He received 'Flinders University Rural Health SA Excellence in Research Award - 2019 from the College of Medicine \& Public Health at Flinders University. He is well-published in high impact journals.

Anita De Bellis Dr Anita De Bellis is a Senior Lecturer in the College of Nursing and Health Sciences at Flinders University of South Australia. Anita has had many years of experience in aged care nursing across a number of contexts in practice, research and teaching. In practice she has worked in different settings in both the acute, community and aged care sectors. Since becoming a nurse academic, the focus of her teaching and research has been in aged care and nursing practice across sectors especially in areas of dementia care and end of life care.

Wendy Abigail Dr Wendy Abigail is a RN, Senior Lecturer within the College of Nursing and Health Sciences at Flinders University with full academic status. Her skills and expertise are directly relevant to women's health research as well as grey nomad research projects due to her strong history of work in these areas. Women's health (including sexual and reproductive health [SRH], breast cancer, women's empowerment) is her area of research focus with expertise in mixed methods research including quantitative methods (trend analysis and descriptive methods) qualitative research and case study methodology. Additionally, she has a strong research interest in gey nomad research, with a focus on diabetes heatlh. Dr Abigail leads, has led or is Associate Chief Investigator for research grants with outputs including peerreviewed journal articles, national and international conference presentations including being an invited speaker, as well as non-peer reviewed publications and media engagements locally and internationally. Dr Abigail is recognised nationally and internationally by clinicians, practitioners and researchers for her work and collaborations in the fields of women's health and in the field of scholarship in teaching and learning. 